\section{Condensing Osteitis of the Clavicle}

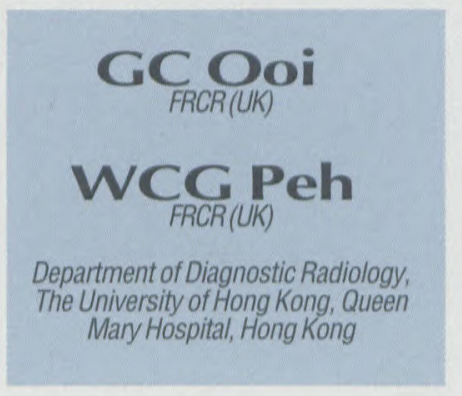

\section{Abstract}

\section{Condensing osteitis of the clavicle}

is a rare benign condition of

unknown aetiology which afflicts

women of child-bearing age. It is

characterised radiologically by

sclerosis of the infero-medial

aspect of the clavicle, with

preservation of the sternoclavicular

joint in the early stages of the

disease. The clinico-radiological

features of this condition are

discussed in this review, together

with other conditions which

resemble this entity.

\section{Introduction}

Condensing osteitis of the clavicle is a rare benign but painful disorder of unknown by sclerosis of the medial end of the clavicle, without involvement of the sternoclavicular joint. This term is actually a misnomer, as inflammation is not recognised to be a component of this disorder. ${ }^{1}$ Jurick (1994) in a recent comprehensive review of the literature, found 62 reported cases of what she termed "noninflammatory sclerosis of the clavicle", in addition to 14 of her own cases. ${ }^{2}$ Besides "condensing osteitis", other descriptive terms used include "Friedrich's disease",, 4 "post-traumatic reactive and resorptive lesions of the medial end of the clavicle", "non-inflammatory sclerosis" and simply, "sclerotic changes" of the sternal end of the clavicle. This paper aims to review the clinisimilar entities.

\section{Condensing osteitis}

Condensing osteitis of the clavicle was first identified as a distinct entity by Brower et al (1974). ${ }^{7}$ Their original description of the clinical, radiographic and histological features of this lesion, with symptomatic relieffollowing surgical excision in two young women, has subsequently been recognised by several other workers. To date, about 30 cases of condensing osteitis, mainly in the aetiology. It is characterised radiographically $\mathrm{cal}$ and imaging features of patients with condensing osteitis of the clavicle and other form of case reports, have been documented in English literature ${ }^{1,27-22}$ Nearly all affected patients were adult women (96\%) aged between 26 to 69 years (mean age of 45 years). The majority complained of pain and/or swelling of the medial end of the clavicle. In some cases, the pain radiated to the supraclavicular fossa and ipsilateral shoulder, aggravated by abduction or forward movement of the shoulder. The pain was usually mild but could be severe, with duration of symptoms varying from 4 weeks to 5 years. In about half of the reported cases, there was an association with minor stress or repetitive physical activity, such as lifting of heavy weights or sportsrelatedactivity. 1,27-22 VanHolsbeek(1992) postulated that the clavicular sclerosis may be secondary to chronic abrasion of a spontaneously subluxing stemoclavicular joint. ${ }^{20}$

The radiographic features are characteristic, comprising dense sclerosis at the inferior aspect of the medial clavicle, often associated with mild bony expansion. A hook-like osteophyte arising from the infero-medial aspect of the clavicle has also been recognised as a feature of this condition (Figure 1).The sternoclavicular joint space is not narrowed, at least in the early

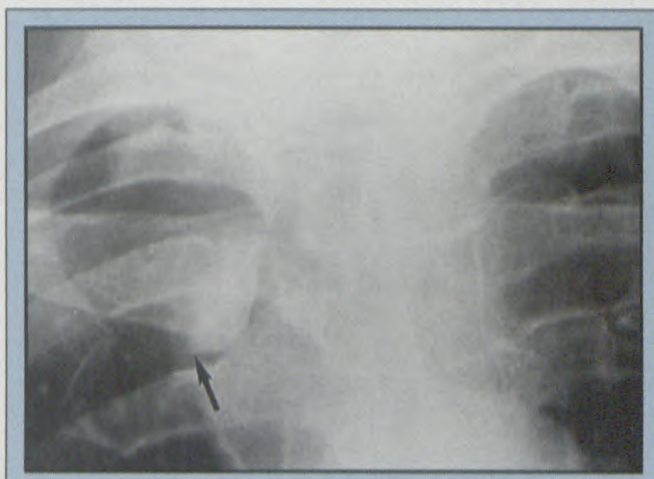

Figure 1: 70-year old woman with dense sclerosis of the right medial clavicle. Note associated inferior osteophyte (arrowed). The left clavicle is normal.

stages of the disease, with preservation of the articular surfaces. Periosteal reaction and bony destruction are absent. Jurick (1994) in a follow-up study of 11 patients with condensing osteitis, demonstrated 


\section{Condensing Osteitis of}

the Clavicle

regression of bony sclerosis and development of adjacent osteoarthritis with time. ${ }^{2}$ Computed tomography (CT) depicts accurately the extent and nature of bone involvement. It demonstrates the preservation of the sternoclavicular joint, the sclerotic involvement of the sternal end of the clavicle and excludes a destructive or aggressive process. The bonemarrow cavity is typically obliterated, with soft tissue swelling occasionally reported(Figures 2 and 3). ${ }^{111,12,14-20}$ These lesions also produce

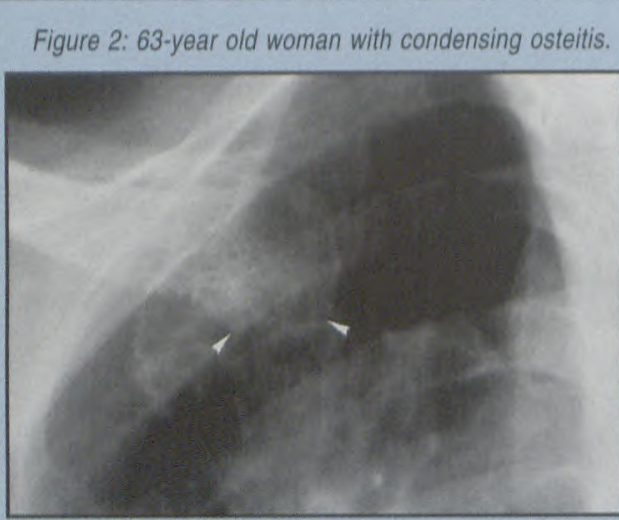

(a) Radiograph shows typical distribution of sclerosis in a slightly expanded medial clavicle (arrowheads). CT taken at contiguous levels demonstrates

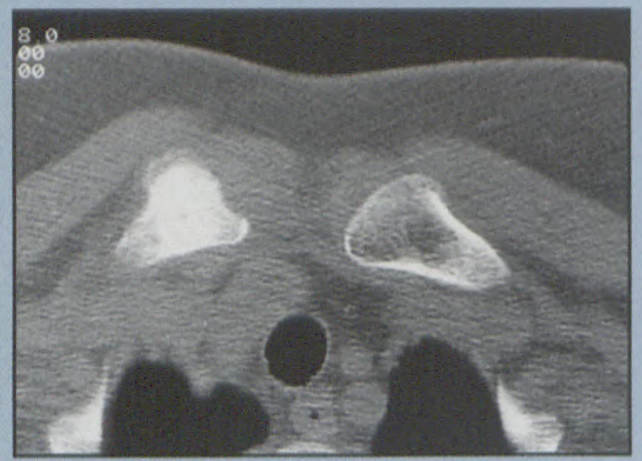

(b) dense homogeneous sclerosis of the right inferomedial clavicle and

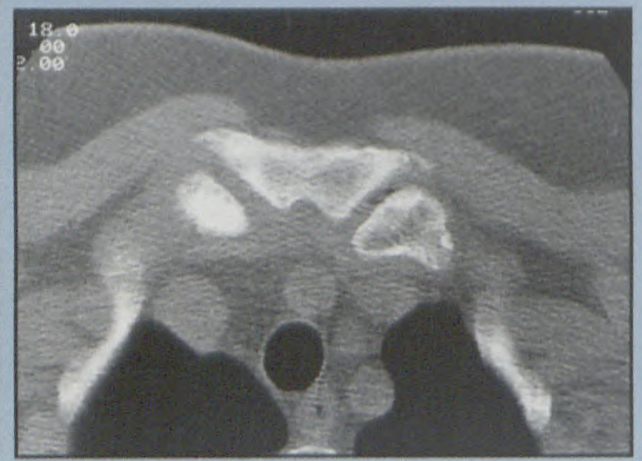

(c) preservation of the adjacent sternoclavicular joint.

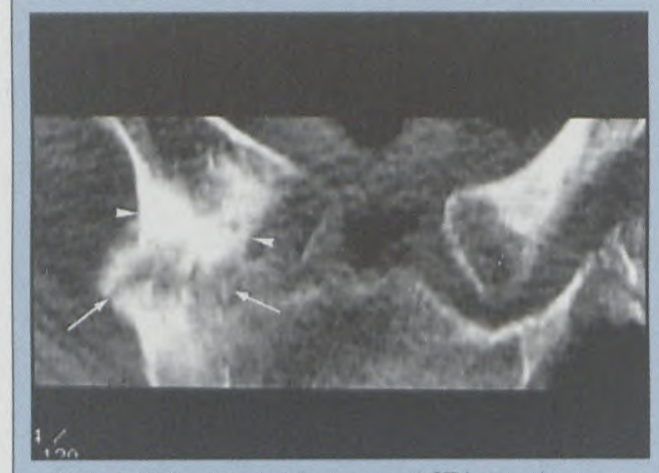

Figure 3. Reformatted oblique coronal CT image demonstrates typical site of clavicular sclerosis (arrowheads), as well as degenerative changes at its articulation with the calcified right first costal cartilage (arrowed). The sternoclavicular joint is relatively unaffected.

markedly increased focal tracer uptake on isotope bone scans, corresponding to the site of bony sclerosis (Figure 4). . $^{1,-17,19,21}$ Magnetic resonance imaging is useful in

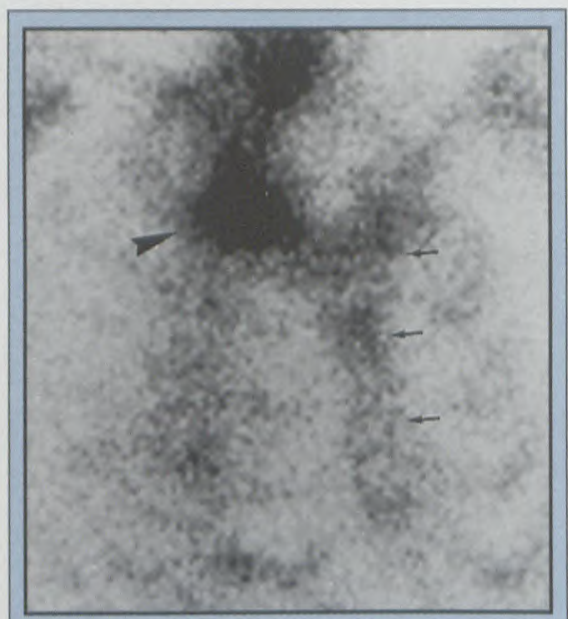

Figure 4: Isotope bone scan (Tc-99m methylene diphosphonate, 2 hour delay) in a 67-year old woman with condensing osteitis. Right anterior oblique projection of the chest shows an intense area of increased uptake at the medial end of the right clavicle (arrowhead). The sternum is arrowed.

confirming the benign nature of the clavicular sclerosis, demonstrating areas of hypointense signal intensities on both spinecho $\mathrm{Tl}$ and 2 weighted images ${ }^{21,23}$ which represent the regions of sclerotic bone. The histological features of condensing osteitis are an increased amount of normal bone with marrow obliteration, thickened cancellous bone and trabecular reinforcement. ${ }^{1,711,14-17,19,23}$ Some authors have also reported marrow fibrosis and osteonecrosis, suggesting a low-grade ischaemic process. ${ }^{1,14,17,23}$ The true cause of condensing osteitis is unknown, although it is generally accepted as representing a response to mechanical stress which particularly affects women of child-bearing age.

Symptomatic patients should be managed with nonsteroidal anti-inflammatory drugs and limitation of physical activities. If the diagnosis is in doubt or if the patient is refractory to conservative treatment, excision of the medial end of the clavicle is recommended. ${ }^{1,11,12,15}$

\section{Friedrich's disease}

Friedrich's disease is ischaemic necrosis of the medial clavicular epiphysis. It is a rare disorder, with only 28 cases having been reported to date.$^{2-4}$ Clinically, the presenting signs and symptoms are similar to that of condensing osteitis. There is painful swelling over the sternoclavicular joint, which may be aggravated by movement. Unlike condensing osteitis, however, Friedrich's disease is not confined to the adult population, and in fact typically afflicts children and adolescents. The radiological appearances may resemble those of condensing osteitis but careful examination of plain radiographs will demonstrate that the entire medial end of the clavicle is sclerotic, with fragmentation and a notch-like defect at the articular surface. Theselesions appear hot on isotope bone scans, hence are scintigraphically indistinguishable from condensing osteitis. The disease is often self limiting with eventual normalisation of radiographic appearance after conservative treatment. Necrosis, with fibrosis of the marrow cavity and empty lacunae, is the main histological feature of Friedrich's disease. Trauma or an embolic event at the medial clavicular epiphysis is believed to cause the ischaemic necrosis that is the hallmark of this condition. ${ }^{24}$ 


\section{Other lesions which may resemble condensing osteitis}

Chronic recurrent osteomyelitis is an unusual condition usually affecting children. With clavicular involvement, development of hyperostosis and bony enlargement may mimic condensing osteitis. ${ }^{23}$ Appell et al (1983) used the term condensing osteitis to describe marked sclerotic enlargement of the medial two-thirds of the clavicle associated with periosteal reaction in seven girls (aged 7 to 15), three of whom had multifocal bone involvement. All cases had raised erythrocyte sedimentation rates, two had high white cell count while four responded to antibiotics. ${ }^{24}$ Jones $e t$ al (1990) described radiographic findings of marked expansion and sclerosis of the medial two-thirds of the clavicle, with thick dense periosteal reaction, in 3 children. Bone biopsies confirmed chronic osteomyelitis in one patient and non-specific inflammatory changes in the other two. The patients responded to antibiotics leading to the authors to suggest that condensing osteitis could be a form of low grade osteomyelitis. ${ }^{25}$ The consensus in subsequent reviews was that these authors reported cases of chronic recurrent multifocal osteomyelitis, which differed clinically and radiologically from condensing osteitis. ${ }^{1,17,23,26}$

Apter et al (1992) described posttraumatic reactive and resorptive lesions of the medial end of the clavicle in 3 young soldiers, biopsy of which showed new bone formation and chronic inflammation. These changes were thought to be stress-related. ${ }^{5}$ Sternocostoclavicular hyperostosis is another condition which typically affects the medial end of the clavicle, as well as other juxtasternal structures. Unlike condensing osteitis, it afflicts older male patients who present with bilateral painful anterior chest wall swellings and pustular lesions of the palms and soles. About 200 cases have been reported to date. ${ }^{27}$ Other differential diagnoses of medial clavicular lesions which may be considered include sternoclavicular joint pyoarthrosis, septic arthritis and osteoarthritis, and miscellaneous conditions like osteoid osteoma and fibrous dysplasia. ${ }^{1,23}$

\section{Summary}

Despite some similarity in presenting signs and symptoms, with careful radiological assessment one should be able to differentiate condensing osteitis from Friedrich's disease and the other conditions that resemble this entity. The selective sclerotic involvement of the inferomedial aspect of the clavicle in women of child-bearing age, in the absence of constitutional symptoms, should lead one to the correct diagnosis. Recognition of this condition should spare the patient from further unnecessary and potentially invasive investigations.

\section{References}

1. Greenspan A Gerscovich E, Szabo RM et al. Condensing osteitis of the clavicle: a rare but frequently misdiagnosed condition. American Journal of Roentgenology 1991;156:1011-1015.

2. Jurick AG. Noninflammatory sclerosis of the sternal end of the clavicle: a follow-up study and review of the literature. Skeletal Radiology 1994;23:373-378.

3. Levy M, Goldberg I, Fischel RE et al. Friedrich's disease. Aseptic necrosis of the sternal end of the clavicle. Joumal of Bone and Joint Surgery 1981;63B:539-541.

4. Christensen $\mathrm{PB}$ and Christensen I. A case of Friedrich's disease of the clavicle. Acta Orthopaedica Scandinavica 1987;58:585-586.

5. Apter S, Hertz M, Salai M et al. Post-traumatic reactive and resorptive lesions of the medial end of the clavicle. Clinical Imaging 1992;16:40-42.

6. Jurick AG, de Carvalho A, Graudal H. Sclerotic changes of the sternal end of the clavicle. Clinical Radiology 1985;36:23-25.

7. Brower AC, Sweet DE, Keats TE. Condensing osteitis of the clavicle: a new entity. American Journal of Roentgenology 1974;121:17-21.
8. Teates CD, Brower AC, Williamson BRJ et al. Bone scans in condensing osteitis of the clavicle. Southern Medical Joumal 1978;71:736-738.

9. Simpson AJ. Positive bone scintigraphy in condensing osteitis of the clavicle. Clinical Nuclear Medicine 1978:3:204.

10. Duro JC Estrada P, Ribas D et al. Condensing osteitis of the clavicle. Arthritis and Rheumatism 1981;24:14541455.

11. Cone RO, ResnickD, Goergen TG et al. Condensing osteitis of the clavicle. American Joumal of Roentgenology 1983;141:387-388.

12. Weiner SN, Levy M, Bernstein Ret al. Condensing osteitis of the clavicle. Journal of Bone and Joint Surgery 1984;66A:1484-1486

13. Hamilton-Wood C, Hollingworth P, Dieppe Pet al. The painful swollen sterno-clavicular joint. The British Joumal of Radiology 1985;58:941-945

14. Franquet T, LecumberriF, Rivas A et al. Condensing osteitis of the davide. Skeletal Radiology 1985;14:184-187.

15. Kruger GD, Rock MG, Munro TG. Condensing osteitis of the clavicle. Joumal of Bone and Joint Surgery 1987;69A:550-557.

16. Stewart CA, Siegel ME, King D et al. Radionuclide and radiographic demonstration of condensing osteitis of the clavide. Clinical NudearMedicine 1988;13:177-178.

17. Outwater E, Oates E. Condensing osteitis of the clavicle: case report and review of the literature. Journal of NudearMedicine 1988;29:1122-1125,

18. Smith J, Yuppa F, Watson RC. Primary tumors and tumor-like lesions of the clavicle. Skeletal Radiology 1988,17:235-246

19. Abdelwahab IF, Hermann G, Ramos R et al. Case report 623. Skeletal Radiology 1990;19:387-389.

20. Van Holsbeek M, Van Melkebeke J, Dequeker J et al. Radiographic findings of spontaneous subluxation of the sternoclavicular joint. Clinical Rheumatology 1992;11:376-381.

21. Vierboom MAC, Steinberg JDJ, Mooyaart EL et al. Condensing osteitis of the clavicle: magnetic resonance imaging as an adjunct method for differential diagnosis. Annals of the Rheumatic Diseases 1992;51:539-541.

22. Latifi HR, Gilula LA. Imaging rounds. Orthopaedic Review 1992;21:767-774.

23. Resnick D. Endocrine diseases. In: Resnick Ded. Diagnosis of bone and joint disorders, 3rd ed. Philadelphia: WBSaunders Co, 1995.

24. Appell RG, Oppermann HC, Becker W et al. Condensing osteitis of the clavicle in childhood: a rare sclerotic bone lesion. Pediatric Radiology 1983;13:301-306.

25. Jones MW, Carty H, Taylor JF et al. Condensing osteitis of the clavicle: does it exist? Joumal of Bone and Joint Surgery 1990;72B:464-467.

26. Kerr R, Resnick D. Condensing osteitis of the clavide. Joumal of Bone and Joint Sungery 1992;74B:163-164.

27. Saghafi M, Henderson MJ, Buchanan WW. Sternocostoclavicular hyperostosis. Seminars in Arthritis and Rheumatism 1993;22:215-223. 